\title{
Síndrome Lipodistrófica do HIV: Um Novo Desafio para o Endocrinologista
}

$\mathrm{C}$ ERCA DE 42 MILHÕES DE PESSOAS estão infectadas pelo HIV no mundo (1). A introdução da terapia anti-retroviral de alta potência (bighly active antiretroviral therapy - HAART), principalmente em indivíduos jovens e crianças, aumentou muito a expectativa de vida. No entanto, temse observado com maior freqüência uma síndrome caracterizada por redistribuição anormal da gordura corporal, alterações no metabolismo glicêmico, resistência à insulina e dislipidemia, conhecida como síndrome lipodistrófica do HIV (SLHIV). Os pacientes apresentam perda de gordura subcutânea na face, braços, pernas e nádegas e acúmulo de gordura no pescoço e abdômen. Tais alterações podem determinar um significativo risco cardiovascular na população soropositiva sob HAART. Além disso, estudos demonstram redução na qualidade de vida dos pacientes, decorrente das alterações corporais lipodistróficas, limitando a aderência ao tratamento.

O surgimento dessa nova entidade nos remete às formas clássicas de lipodistrofia não associadas ao HIV, como as lipodistrofias congênitas ou adquiridas parciais ou generalizadas. Essas condições de baixa prevalência são associadas a complexas anormalidades metabólicas de difícil tratamento. A SLHIV tem uma prevalência entre 30 e $50 \%$ (2) e relaciona-se ao uso dos anti-retrovirais (ARV), em especial ao grupo dos inibidores de proteases (IP). O risco de desenvolver essa síndrome aumenta com a duração do tratamento, a idade do paciente e o nível de imunodeficiência.

No artigo de Guimarães e cols. (3), publicado neste número dos "Arquivos", são avaliados dados antropométricos, os perfis lipídico e glicêmico de pacientes infectados pelo HIV, tratados ou não com ARV. Os autores evidenciam, nos pacientes infectados pelo HIV usuários de HAART, aumento dos níveis de triglicérides, colesterol total, glicemia e insulina, caracterizando resistência à insulina.

Essas alterações têm muita similaridade com os achados mostrados em uma recente publicação (4), na qual foram estudados 788 adultos infectados pelo HIV sob HAART e avaliados pelos critérios da International Diabetes Foundation (IDF) e do U.S. National Cholesterol Education Program Adult Treatment Panel III (NCEP-ATP III). A prevalência da síndrome metabólica neste grupo foi de 14\% pelos critérios da IDF e 18\% pelos critérios da ATP III. Metade dos pacientes apresentava pelo menos duas características da síndrome metabólica, mas não foram classificados como tal porque a medida da cintura ou a razão cintura/quadril não estavam nas faixas da síndrome metabólica definidas por esses critérios. A prevalência de diabetes tipo 2 foi 5 a 9 vezes maior no subgrupo dos pacientes com síndrome metabólica. $\mathrm{O}$ mesmo grupo havia mostrado níveis diminuídos de adiponectina em pacientes HIV com lipodistrofia (5). Nesse estudo, adiponectina foi $20 \%$ menor nos pacientes com síndrome metabólica. Esse dado não surpreende, já que a obesidade visceral é associada com hipoadiponectinemia. Além disso, esses pacientes com HIV, SM e lipodistrofia apresentam também aumento da proteína C-reativa e hiperleptinemia. A SM estudada nesses pacientes foi mais freqüente naqueles

\section{editorial}

\section{ORSINE VALENTE \\ Angélica M. Martins Valente}

Prof. Adjunto de Medicina de Urgência, Universidade Federal de São Paulo, e Prof. Adjunto Disciplina de Endocrinologia, Faculdade de Medicina do ABC. (OV) Pós-graduanda Disciplina de Endocrinologia, Universidade Federal de São Paulo, SP. (AMMV) 
pacientes recebendo IP. Embora os mecanismos pelos quais os IP causem lipodistrofia permaneçam pouco esclarecidos, postula-se que essas drogas interfiram na diferenciação de pré-adipócitos (6). Estudos em biópsias de pacientes com lipodistrofia que estavam recebendo IP evidenciaram leve a moderada apoptose do adipócito subcutâneo (7).

As alterações metabólicas da SLHIV dos pacientes sob HAART, principalmente os IP, também foram descritas em revisão recente sobre o tema publicada nos "Arquivos" (8).

As principais causas de morbidade e mortalidade nos estados lipodistróficos do HIV são a hipertrigliceridemia, a hipercolesterolemia, a esteatose hepática prolongada, com possibilidade de evolução para a cirrose e o diabetes com suas complicações de longo prazo. Em pacientes com hipertrigliceridemia que persiste mesmo após mudanças da dieta e exercício físico, deve-se introduzir tratamento com fibrato. Nos pacientes com hipercolesterolemia, a pravastatina, a rosuvastatina e a ezetimiba são medicamentos úteis, já que a sinvastatina, lovastatina e atorvastatina são metabolizadas pelo citocromo P450 CYP3A4, inibido pelos IPs. Com o aparecimento do diabetes, além das medidas dietéticas e do aumento da atividade física, pode-se necessitar de um hipoglicemiante oral ou insulina para prevenir as complicações da hiperglicemia a longo prazo. A metformina, além de reduzir o apetite, induz perda de peso e diminui a esteatose hepática, sendo uma escolha adequada ( 1 ). $\mathrm{O}$ tratamento com pioglitazona, $30 \mathrm{mg}$ uma vez ao dia, por 48 semanas mostrou, em um estudo randomizado, duplo cego, placebo-controlado (9), melhora da sensibilidade à insulina e significante aumento na gordura subcutânea. Além disso, não se observou aumento dos níveis de lipídeos plasmáticos, achado freqüente quando se administra rosiglitazona.

A introdução dessas novas drogas no tratamento da infecção pelo HIV aumentou muito a expectativa de vida desses pacientes, de tal maneira que o co- nhecimento e a avaliação precoce dessas alterações metabólicas e o tratamento adequado seguramente contribuirão para impedir ou diminuir outras co-morbidades, melhorando com isso a qualidade de vida e diminuindo a mortalidade desses pacientes.

\section{REFERÊNCIAS}

1. Garg A. Acquired and inherited lipodystrophies. N Engl J Med 2004;350:1220-34.

2. Beherens G, Schmidt RE. Lipodystrophy syndrome. Available at: <http://www.hivmedicine.com/hivmedicine2006. pdf $>$.

3. Guimarães MMM, Greco DB, Oliveira-Júnior AR, Penido MG, Machado LJC. Distribuição da gordura corporal e perfis lipídico e glicêmico de pacientes infectados pelo HIV. Arq Bras Endocrinol Metab 2007;51(1):42-51.

4. Samaras K, Wand H, Law M, Emery S, Cooper D, Carr A. Prevalence of metabolic syndrome in HIV-infected patients receiving highly active antiretroviral therapy using International Diabetes Foundation and Adult Treatment Panel III criteria. Diabetes Care 2007;30(1):113-9.

5. Carr A, Workman C, Carey D, Rogers G, Martin A, Baker D, et al.; the Rosey Investigators. No effect of rosiglitazone for HIV1 lipoatrophy: a randomized, double-blind, placebo-controlled trial. Lancet 2004;363:429-38.

6. Dowell P, Flexener C, Kwiterovich PO, Lanes MD. Suppression of preadipocyte differentiation and promotion of adipocyte death by HIV protease inhibitors. J Biol Chem 2000;275:41325-32.

7. Domingo P, Matias-Guiu X, Pujol RM, Francia E, Lagarda E, Sambeat MA, et al. Subcutaneous adipocyte apoptosis in HIV-1 protease inhibitor-associated lipodystrophy. AIDS 1999;13:2261-7.

8. Valente AMM, Reis AF, Machado DM, Succi RCM, Chacra AR. Alterações metabólicas da síndrome lipodistrófica do HIV. Arq Bras Endocrinol Metab 2005;49:871-81.

9. Slama $L$, Lanoy $L$, Valentin MA, et al. Effect of pioglitazone on HIV-1 related lipoatrophy: a randomized doubleblind placebo-controlled trial (ANRS 113) with 130 patients. $13^{\text {th }} \mathrm{CROI}, 2006$, Denver, USA (Abstract 151LB).

Endereço para correspondência:

Orsine Valente

R. Pedro de Toledo 598

04039-001 São Paulo, SP

Fax: (1 1 ) 5575-2970

E-mail: orsine.ops@terra.com.br 